\title{
Aprovechamiento forestal de manglares en el estado de Nayarit, costa Pacífica de México
}

\author{
Juan Ignacio Valdez Hernández ${ }^{1}$
}

\begin{abstract}
RESUMEN
Se presenta un estudio del efecto de la cosecha de madera en la estructura y repoblación natural de manglares del Ejido Villa Juárez, Nayarit. Los resultados indican que, con excepción de la densidad y área basal de Laguncularia racemosa pertenecientes a la categoría diamétrica de $18 \mathrm{~cm}$, en donde se detectaron diferencias significativas entre rodales cosechados y no cosechados, la estructura de los manglares en el área de estudio fue similar. Si bien la cantidad de renuevos de Avicennia germinans mayores que $1.3 \mathrm{~m}$ fue la única clase de altura que tuvo diferencias significativas, se presentó una mayor y más continua abundancia de la repoblación en rodales cosechados de Laguncularia que en los no cosechados.
\end{abstract}

PALABRAS CLAVE:

Avicennia germinans, cosecha de madera, estructura del rodal, Laguncularia racemosa, regeneración natural.

\begin{abstract}
The effect of wood harvest on the structure and natural repopulation of mangroves from Ejido Villa Juarez, Nayarit was studied. The results indicate that, with exception of the density and basal area of Laguncularia racemosa corresponding to the $18 \mathrm{~cm}$ diameter category, in which significative differences between harvested and notharvested stands were detected, the structure of the mangroves in the area was similar. Although the amount of sprouts of Avicennia germinans greater than $1.3 \mathrm{~m}$ was the only height class that had significative differences, it was found a larger and more continuous abundance of repopulation in harvested stands of Laguncularia than in those not harvested.
\end{abstract}

KEY WORDS:

Avicennia germinans, wood harvest, stand structure, Laguncularia racemosa, natural regeneration. 


\section{INTRODUCCIÓN}

Desde un punto de vista tanto biológico como socioeconómico, los manglares constituyen uno de los ecosistemas costeros más importantes de México. Estos proporcionan una amplia variedad de recursos naturales, bienes y servicios, entre los cuales se encuentran medicinas, leña, taninos y material de construcción, así como lugares para la protección y desarrollo de fauna acuática y terrestre con fines de uso doméstico, comercial o de conservación.

La cosecha de madera de especies de mangles, con un volumen superior a los $100000 \mathrm{~m}^{3}$ para México en 1991 (Velázquez et al., 1995), es otro de los productos que representan un beneficio para las comunidades humanas establecidas en las zonas costeras, sea mediante su venta o por consumo de subsistencia. Poco más del $40 \%$ del volumen de madera antes mencionado se produjo en el estado de Nayarit, en donde tallos de especies de mangles, especialmente para la construcción de galeras de secado de la hoja del tabaco, se han cosechado desde la década de 1960, previa autorización oficial, por al menos 13 ejidos asentados en la planicie costera de dicho estado.

Debido a notificaciones realizadas por organizaciones locales no ejidales, quienes consideraron los volúmenes de madera cosechados como muy elevados, así como a la propia opinión de la delegación de SEMARNAP en el estado por la protección ecológica de estos ambientes, a partir de 1994 no se autorizaron permisos para el aprovechamiento forestal de manglares en Nayarit. Esta situación afectó en gran medida la economía de las familias que dependen durante un tercio del año de la cosecha de madera de mangles y ocasionó cortas ilegales en un gran número de localidades.

Con el deseo de generar parte de la información para una adecuada toma de decisiones respecto a la conservación de estos ecosistemas, el propósito de este documento fue conocer la estructura a nivel rodal y la repoblación natural de algunos manglares bajo aprovechamiento maderable en el estado de Nayarit. Para ello se escogió a uno (Villa Juárez) de los trece Ejidos que cortan madera de mangles en el estado, tomando como base una mayor antigüedad y permanencia del mismo en esta actividad, así como su volumen de madera reportado.

No obstante que la estructura de manglares a nivel rodal ha sido descrita por Pool et al. (1977) en Norte y Centro América, Cintron et al. (1985) en Puerto Rico, Lahmann et al. (1987) en Ecuador y Silva et al. (1991) en Brasil, con énfasis en su densidad y área basal; así como por Jiménez (1988, 1990) en Costa Rica, Robertson et al. (1991) en Papua Nueva Guinea y Saifullah et al. (1994) en Pakistán, con atención a la distribución de individuos por categoría diamétrica; son escasos los estudios que, como el de Sukardjo (1987) en Indonesia, han analizado la estructura de los manglares desde una perspectiva de uso y manejo forestal. De la misma forma, y con excepción de los trabajos de Srivastava y Daud (1978) en Malasia y de Alrasjid y Effendi (1986) en Indonesia, no se ha abordado la influencia de la extracción maderable en la repoblación natural de estos bosques.

Esta falta de información es más notoria en México, donde los estudios se han dirigido principalmente a la zonación (Thom, 1967; Vázquez, 1971; López Portillo, 1982), fisonomía (Lot et al., 1975; Menéndez, 1976; Lot y Novelo, 1990; Trejo et al., 1993) y distribución horizontal (Valdez, 1991; Flores, et al., 1992) de los manglares $\mathrm{y}$, en donde, aparte del trabajo de Ramírez (1995), los datos acerca de la repoblación natural de estos bosques son casi inexistentes. Por lo anterior, es necesario llevar a cabo evaluaciones en los manglares de nuestro país que permitan conocer el efecto del corte de madera en su estructura forestal y repoblación natural. 


\section{OBJETIVOS}

Determinar la densidad y área basal de Avicennia germinans y Laguncularia racemosa en rodales sujetos a cosecha de madera, con respecto a rodales no cosechados y cuantificar la abundancia de la repoblación natural en rodales cosechados y no cosechados de ambas especies.

\section{METODOLOGÍA}

\section{Área de estudio}

La presente investigación se realizó en el Ejido Villa Juárez, municipio de Santiago Ixcuintla, estado de Nayarit, hacia la desembocadura y margen derecha del Río Santiago ( $21^{\circ} 38^{\prime} 37^{\prime \prime} \mathrm{N}$, $105^{\circ} 26^{\prime} 25^{\prime \prime}$ )). Esta zona pertenece a la provincia fisiográfica Llanura Costera del Pacífico, subprovincia Delta del Río Grande de Santiago y se distingue por su abundancia en depósitos palustres y aluviales del cuaternario (SPP, 1981). La altitud sobre el nivel del mar varía de $0 \mathrm{~m}$ a $3 \mathrm{~m}$ en la zona de esteros con lagunas y los principales grupos de suelos son Solonchaks y Fluvisols. Las mareas son mixtas semidiurnas con amplitud promedio de $70 \mathrm{~cm}$ (Instituto de Geofísica/UNAM, 1997) y el clima es cálido-subhúmedo con lluvias en verano $\left(A w_{1}(w)\right)$, intermedio en humedad, con temperatura media anual de $26.6^{\circ} \mathrm{C}$ y precipitación media anual de $1267.4 \mathrm{~mm}$ (García, 1987). Los principales tipos de vegetación en la región son manglar, vegetación halófita y selva baja caducifolia.

Los miembros del Ejido Villa Juárez viven en la localidad del mismo nombre, la cual tenía una población de 3070 habitantes en 1990, representando el $3.1 \%$ del total del municipio de Santiago Ixcuintla (INEGI, 1996). Alrededor del $72 \%$ de la población de este municipio se consideró como económicamente activa y casi el $60 \%$ de esta última se dedicó a labores agropecuarias, silvícolas y pesqueras (INEGI, 1996). Este porcentaje es considerablemente mayor que el promedio estatal (38\%) y nacional (20\%) (INEGI, 1997), indicando la importancia de estas actividades como forma de subsistencia en la zona. El Ejido Villa Juárez, fundado en 1945 y con una superficie total de 5 104 ha, cosecha en promedio alrededor de $540 \mathrm{~m}^{3}$ de madera de mangle por año en poco más de 270 ha (INEGl, 1993, 1994, 1998). Este volumen de madera se extrae del bosque en forma de piezas de 7 $\mathrm{cm}$ a $12 \mathrm{~cm}$ de diámetro de las especies Laguncularia racemosa (L.) Gaertn. f. (Combretaceae) y Avicennia germinans (L.) Stearn (Avicenniaceae) y, principalmente, son utilizados en la construcción de galeras para el secado de la hoja del tabaco en el propio municipio de Santiago Ixcuintla.

\section{Métodos}

La vegetación de manglar en el área de estudio está dominada por rodales monoespecíficos de Laguncularia y Avicennia que, no obstante poseer la misma edad ( $\approx 45$ años), algunos de ellos han sido aprovechados con fines maderables: cerca de $2 \mathrm{~m}^{3}$ removidos anualmente por ha, con un total de existencias de 92 $\mathrm{m}^{3} /$ ha a $140 \mathrm{~m}^{3} / \mathrm{ha}$, aplicando un sistema silvícola de selección y un ciclo de corta de 5 a 7 años, en tanto que otros, como parte de un plan de investigación a largo plazo, se han mantenido con escasa o nula intervención humana.

Con objeto de comparar la estructura y repoblación natural de cada especie de mangle entre ambos tipos de rodales, se establecieron en forma aleatoria un total de 20 sitios de medición de $30 \mathrm{~m} \times 10 \mathrm{~m}$ : Diez sitios en rodales de Laguncularia (cinco cosechados, cinco no cosechados) y diez sitios en rodales de Avicennia (cinco cosechados, cinco no cosechados).

Considerando el tipo de información a registrar, cada sitio fue subdividido a su vez en tres parcelas de $10 \mathrm{~m} \times 10 \mathrm{~m}(100$ $\mathrm{m}^{2}$ ), sobre las que se establecieron, en forma aleatoria, dos parcelas de $4 \mathrm{~m} \times 4 \mathrm{~m}$ $\left(16 \mathrm{~m}^{2}\right)$ y cinco de $1 \mathrm{~m} \times 1 \mathrm{~m}\left(1 \mathrm{~m}^{2}\right)$. En las parcelas de $100 \mathrm{~m}^{2}$ se midió el diámetro a la altura del pecho (DAP) de todos los 
individuos mayores a $2.5 \mathrm{~cm}$ de DAP; en las de $16 \mathrm{~m}^{2}$ se registró la densidad de los tallos menores a $2.5 \mathrm{~cm}$ de DAP pero mayores que $1.3 \mathrm{~m}$ de altura; $y$ en las de 1 $\mathrm{m}^{2}$ la abundancia de los tallos menores a $1.3 \mathrm{~m}$ de altura pero mayores que $30 \mathrm{~cm}$ de altura, así como el total de individuos menores a $30 \mathrm{~cm}$ de altura.

El área basal de los tallos mayores a $2.5 \mathrm{~cm}$ DAP fue calculada con la siguiente formula:

$$
B A=\pi / 4(D A P)^{2}
$$

donde:

$$
\begin{aligned}
B A= & \text { área basal en metros cuadrados } \\
D A P= & \text { diámetro a la altura del pecho } \\
& (1.3 \mathrm{~m}) \text { en metros, } \mathrm{y} \\
\pi= & 3.14159
\end{aligned}
$$

La estructura de la población en rodales cosechados y no cosechados, para ambas especies de mangles, fue analizada mediante la agrupación de individuos en categorías diamétricas de 2 $\mathrm{cm}$ (aquellos mayores a $2.5 \mathrm{~cm}$ DAP), de acuerdo con lo sugerido por Pool et al. (1977) y Jiménez (1990) para manglares $\mathrm{y}$, en clases de altura como sigue: plantas $<30 \mathrm{~cm}$ (clase 1); $30 \mathrm{~cm}$ <plantas $<1.3 \mathrm{~m}$ (clase 2); y $1.3 \mathrm{~m}<$ plantas $<2.5 \mathrm{~cm}$ DAP (clase 3 ). Los resultados para el número de individuos, así como para las áreas basales, fueron extrapolados a valores por hectárea y colocados en forma gráfica.

Con objeto de reconocer posibles diferencias significativas entre densidad, área basal y abundancia de la repoblación entre rodales cosechados y no cosechados por especie de mangle, se realizaron análisis de varianza de una vía (ANDEVA) y pruebas de Tukey-Kramer con $p<0.05$. Cuando las variables bajo estudio no cumplieron con la suposición de normalidad en su distribución (prueba de Shapiro-Wilk W), se les aplicó la prueba de Wilcoxon/ Kruskal-Wallis (no paramétrica). Todos los análisis estadísticos se llevaron a cabo en JMP (SAS Institute, 1996).
La distribución del número de individuos por categoría diamétrica fue ajustada mediante ecuaciones de regresión de la forma:

$$
y=\beta_{0}+\beta_{1} \ln (x)
$$

para valores promedio de rodales cosechados y no cosechados en cada especie de mangle.

Los parámetros de regresión se calcularon a través del procedimiento de mínimos cuadrados y la selección de las ecuaciones fue basada en la inspección gráfica del ajuste, dispersión de residuales y valor de $R^{2}$. Se llevaron a cabo análisis de varianza de una vía (ANDEVA) tomando en cuenta un valor de $p<0.010$ mejor. Estos análisis también se efectuaron en JMP (SAS Institute, 1996).

Se detectaron diferencias significativas entre ecuaciones, de rodales cosechados y no cosechados, mediante las pruebas de

Paralelismo:

e

$$
y=\beta_{0}+\beta_{1}(x)+\beta_{2}(d m)
$$

Interacción:

$$
y=\beta_{0}+\beta_{1}(x)+\beta_{2}(d m)+\beta_{3}[(x)(d m)]
$$

donde:

$\begin{aligned} d m= & \text { variable indicadora que toma } \\ & \text { valores de } 1 \text { para rodales } \\ & \text { cosechados y de } 2 \text { para rodales no } \\ & \text { cosechados (Seber, 1977) }\end{aligned}$

Finalmente, los valores promedio del número de árboles en rodales cosechados y no cosechados, por especie, fueron ajustados también a una función de distribución de probabilidades Weibull (Clutter et al., 1983): 


$$
f(x)=\frac{c}{b}\left(\frac{x-a}{b}\right)^{c-1} \exp \left[-\left(\frac{x-a}{b}\right)^{c}\right] \quad a \leq x<\propto \quad=0 \text {, de otra manera. }
$$

donde:

$$
\begin{aligned}
& \mathrm{a}=\text { parámetro de posición } \\
& \mathrm{b}=\text { parámetro de escala } \\
& \mathrm{c}=\text { parámetro de forma }
\end{aligned}
$$

La estimación de estos parámetros se llevó a cabo con el sistema WEST, elaborado por Magaña et al. (1992), el cual utiliza diez procedimientos diferentes y selecciona aquel conjunto de estimadores con mejor bondad de ajuste, con base en los resultados de las pruebas Kolmogorov-Smirnov (KS), diferencia entre media observada y media predicha, así como ji-cuadrada de Pearson $\left(X^{2}\right)$ (Torres et al., 1992).

\section{RESULTADOS}

La distribución del número de individuos y área basal es mostrada por categoría diamétrica, tanto para rodales cosechados como no cosechados, en las figuras 1 y 2 para Laguncularia y en las figuras 3 y 4 para Avicennia.

En rodales cosechados de Laguncularia se aprecia, en general, una distribución de individuos por categoria diamétrica similar a la presente en rodales no cosechados (Fig. 1), la cual se corrobora no sólo porque los valores promedio del numero de individuos por categoría diamétrica en esta especie no presentaron, con excepción de la categoría de $18 \mathrm{~cm}$, diferencias significativas $(p>0.05)$ entre rodales cosechados y no cosechados, sino también porque los modelos de distribución diamétrica de estos individuos entre rodales no cosechados (ec. 1) y cosechados (ec. 2) fueron estadísticamente iguales, ya sea mediante la prueba de paralelismo $(F=$ $0.24, p>0.62, n=36)$ o interacción ( $F=$ $0.31, p>0.58, n=36$ ):

$$
y=1233.41-362.752 \ln (x)
$$

$$
\begin{aligned}
R^{2} & =0.54 \\
F & =18.63 \\
p & =0.0005
\end{aligned}
$$

$$
y=990.933-290.366 \ln (x)
$$

$$
\begin{aligned}
R^{2} & =0.40 \\
F & =10.74 \\
p & =0.0047
\end{aligned}
$$

Así mismo, los valores promedio de densidad en Laguncularia se ajustaron a una distribución Weibull, tanto para rodales no cosechados (ec. 3) como cosechados (ec. 4), habiendo obtenido la estimación más robusta de los parámetros con el procedimiento de percentiles y momentos:

$$
\begin{aligned}
& f(x)=\frac{1.9871}{8.2855}\left(\frac{x-0}{8.2855}\right)^{1.9871-1} \exp \left[-\left(\frac{x-0}{8.2855}\right)^{1.9871}\right] \\
& f(x)=\frac{2.3858}{9.9613}\left(\frac{x-0}{9.9613}\right)^{2.3858-1} \exp \left[-\left(\frac{x-0}{9.9613}\right)^{2.3858}\right]
\end{aligned}
$$


No cosechados

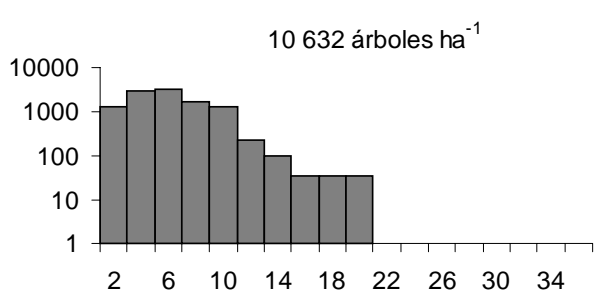

6166 árboles ha $^{-1}$
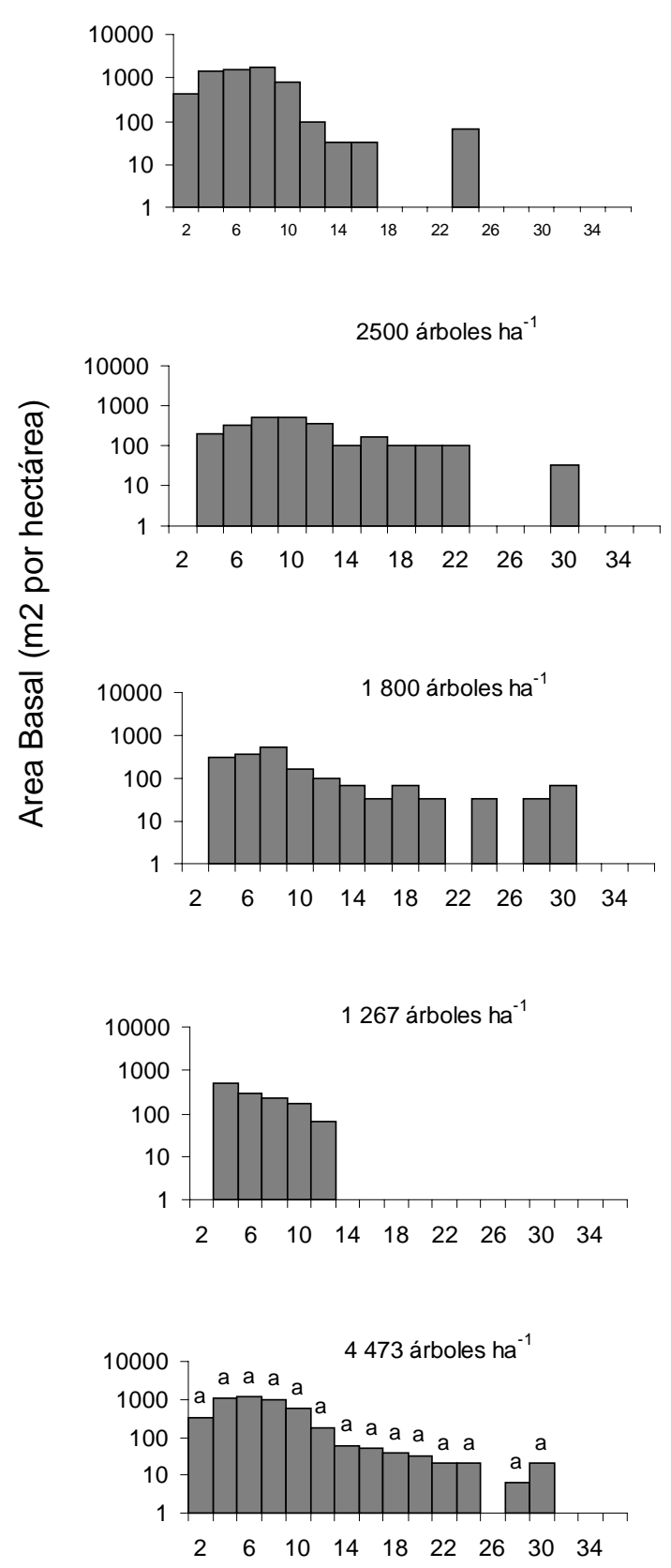

Cosechados

8132 árboles ha $^{-1}$
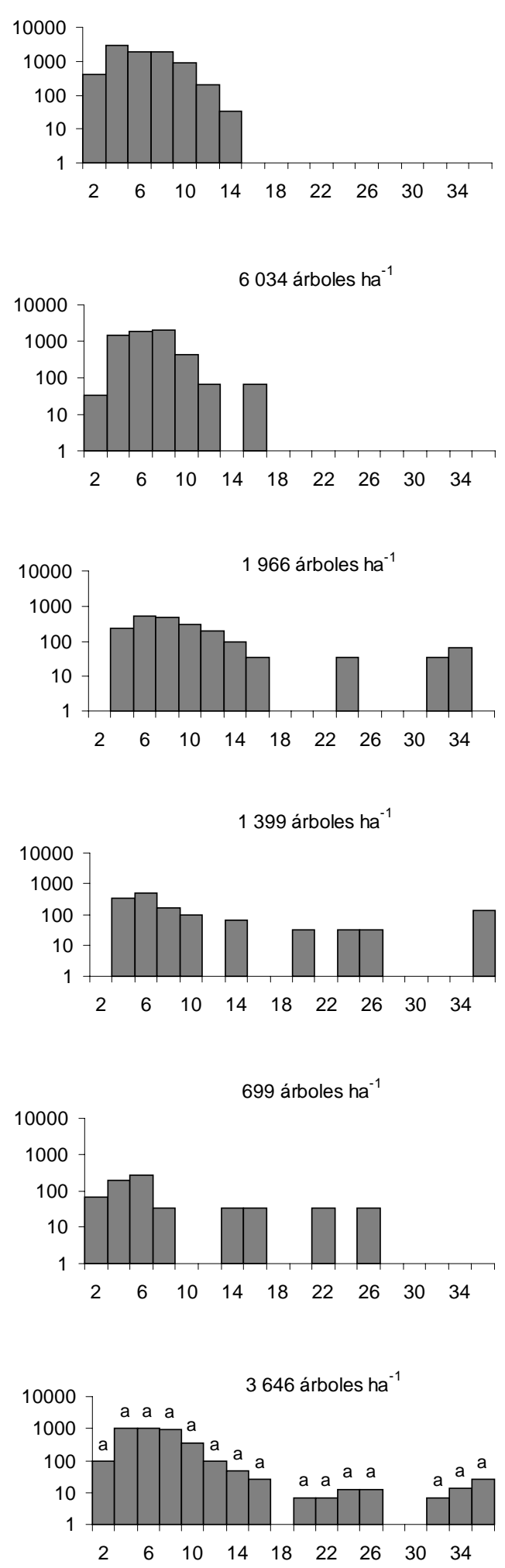

Diámetro a la altura del pecho (DAP) en cm

Figura 1. Distribución por categoría diamétrica del número de árboles por hectárea en rodales no cosechados y cosechados de Laguncularia racemosa en el Ejido Villa Juárez. Letras iguales indican diferencias no significativas $(p>0.05)$ entre rodales cosechados y no cosechados. 
No cosechados
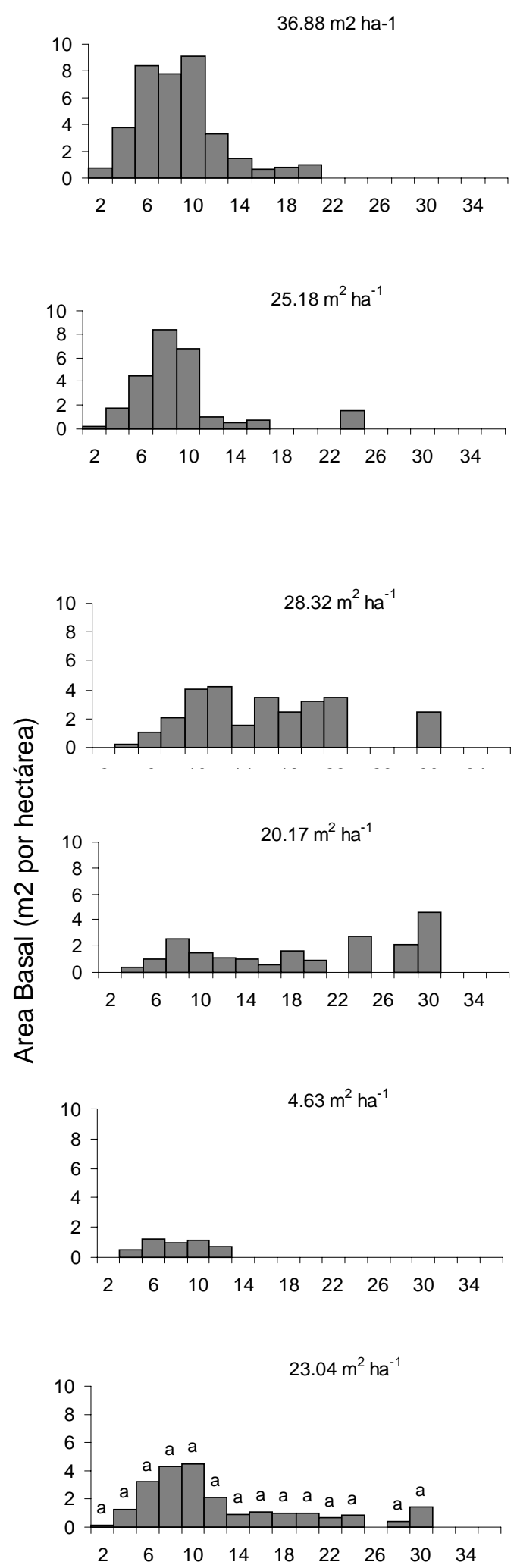

\section{Cosechados}
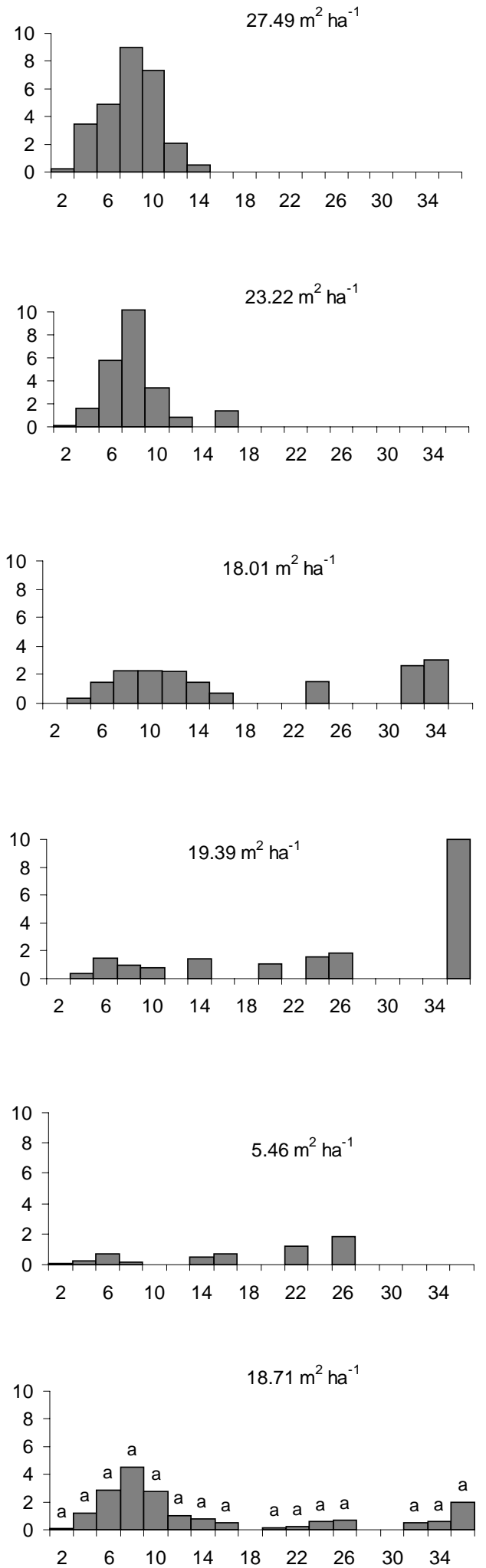

Diámetro a la altura del pecho (DAP) en $\mathrm{cm}$

Figura 2. Distribución por categoría diamétrica del área basal por hectárea en rodales no cosechados y cosechados de Laguncularia racemosa en el Ejido Villa Juárez. Letras iguales indican diferencias no significativas $(p>0.05)$ entre rodales cosechados y no cosechados. 
No cosechados
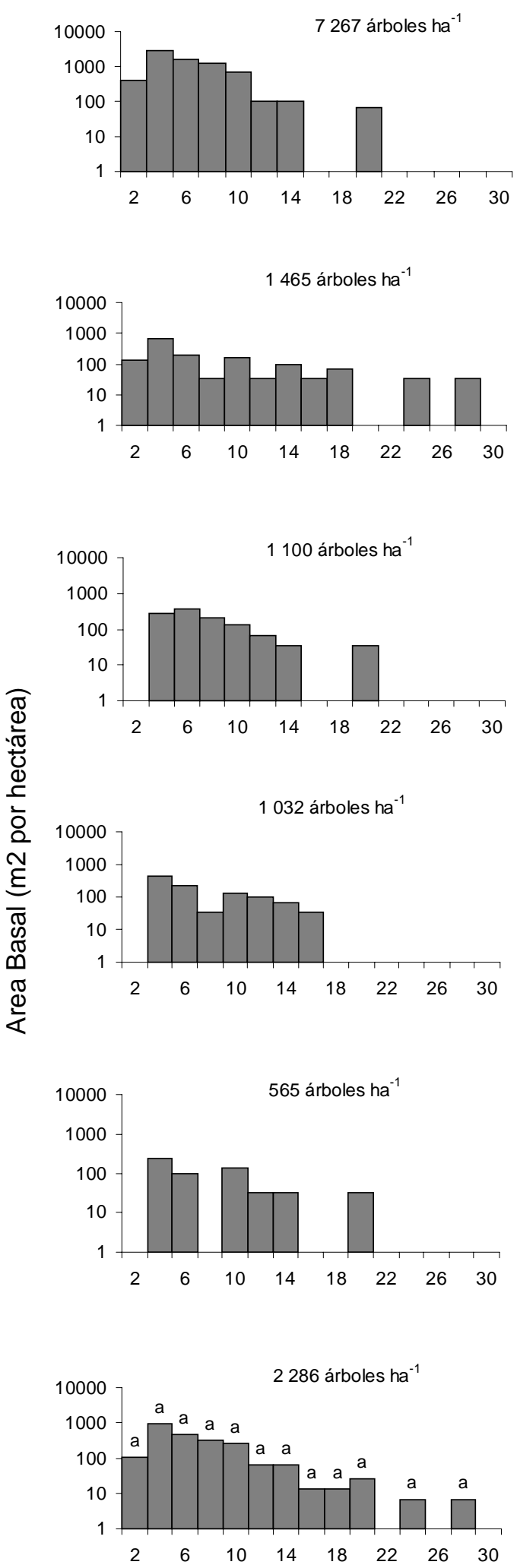

\section{Cosechados}
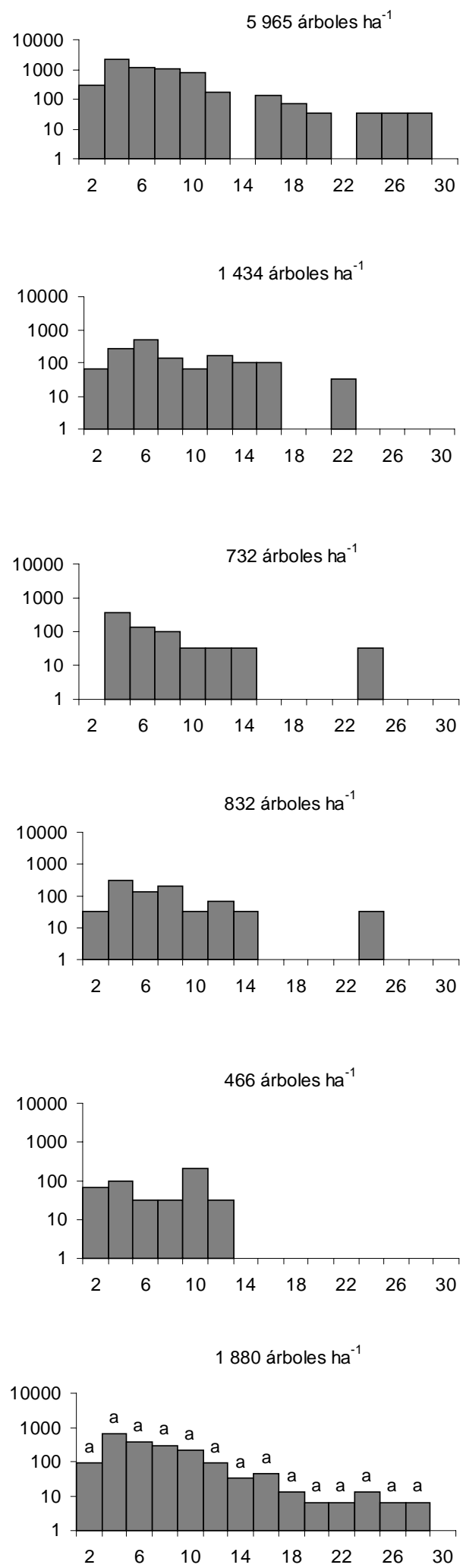

Diámetro a la altura del pecho (DAP) en cm

Figura 3. Distribución por categoría diamétrica del número de árboles por hectárea en rodales no cosechados y cosechados de Avicennia germinans en el Ejido Villa Juárez. Letras iguales indican diferencias no significativas $(p>0.05)$ entre rodales cosechados y no cosechados. 
No cosechados

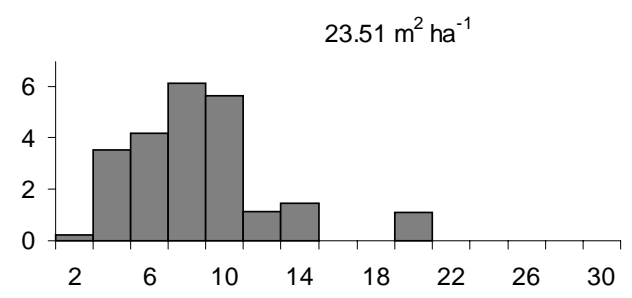

$11.02 \mathrm{~m}^{2} \mathrm{ha}^{-1}$

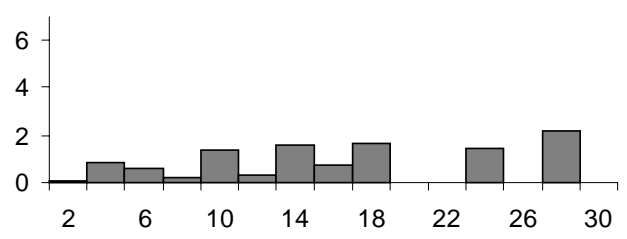

$5.71 \mathrm{~m}^{2} \mathrm{ha}^{-1}$

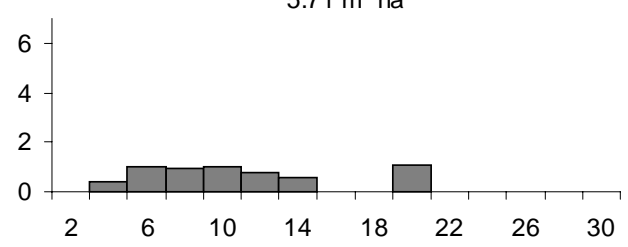

$5.67 \mathrm{~m}^{2} \mathrm{ha}^{-1}$
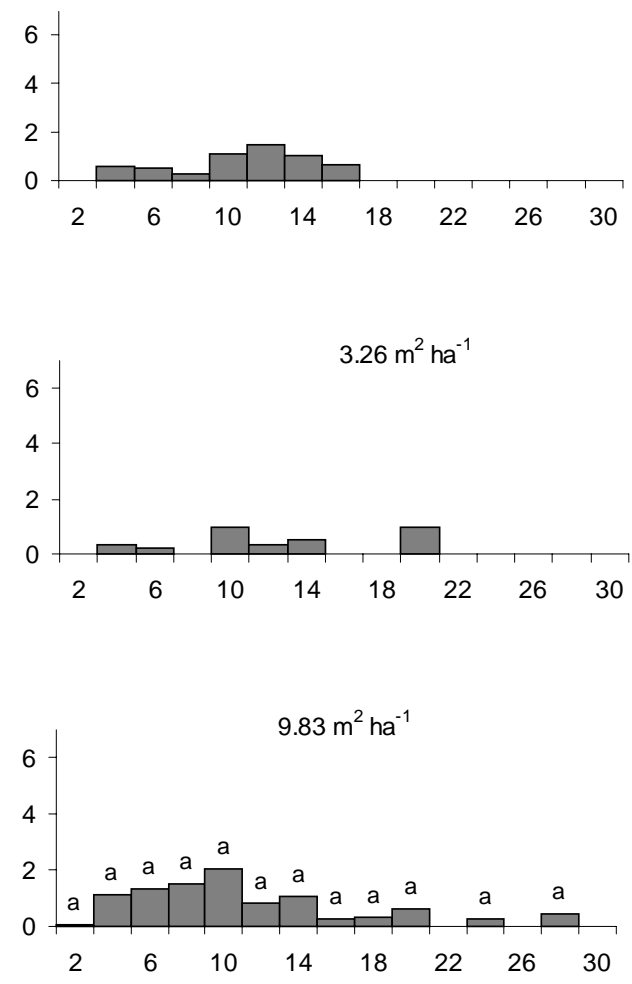

Cosechados

$28.49 \mathrm{~m}^{2} h a^{-1}$
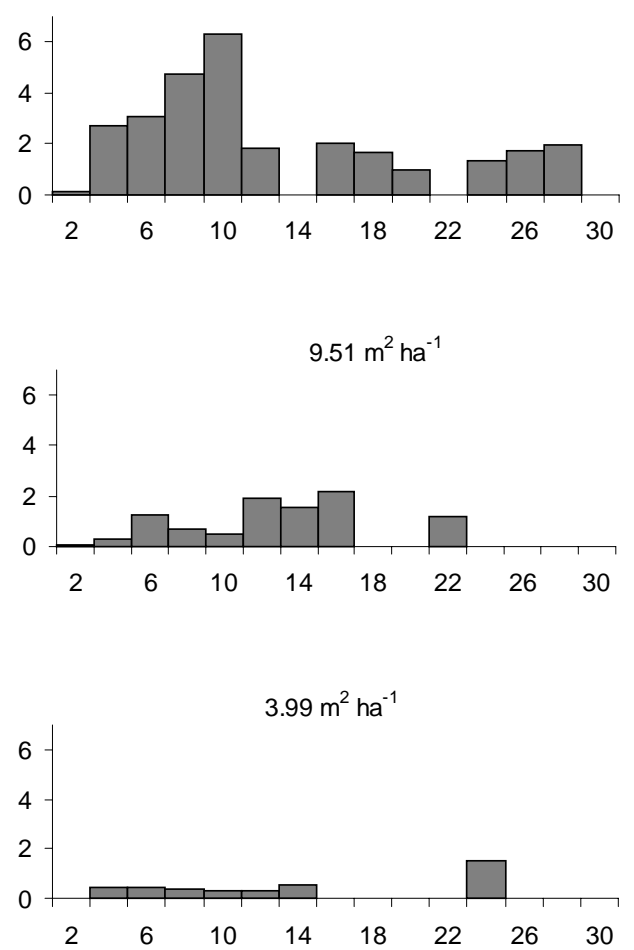

$4.63 \mathrm{~m}^{2} \mathrm{ha}^{-1}$
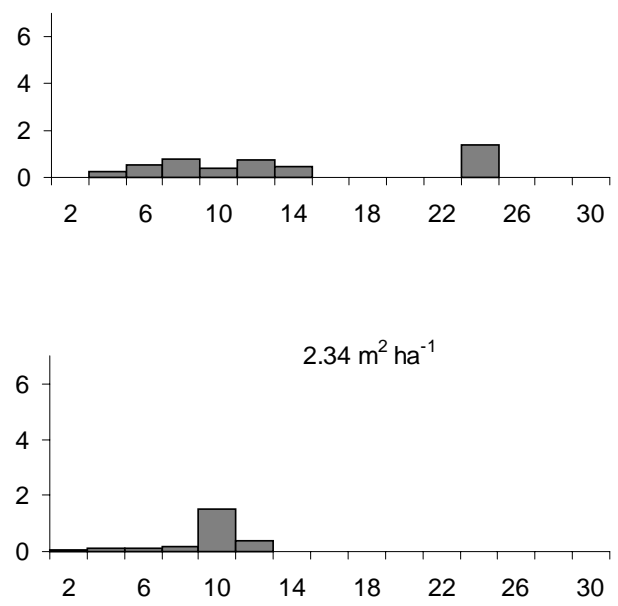

$9.79 \mathrm{~m}^{2} \mathrm{ha}^{-1}$

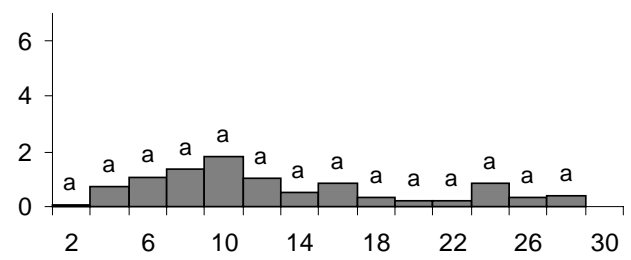

Figura 4. Distribución por categoría diamétrica del área basal por hectárea en rodales no cosechados y cosechados de Avicennia germinans en el Ejido Villa Juárez. Letras iguales indican diferencias no significativas $(p>0.05)$ entre rodales cosechados y no cosechados. 
El área basal de Laguncularia correspondiente a la categoría diamétrica de $18 \mathrm{~cm}$, dimensión de tallos comúnmente utilizada por los pobladores para la construcción de casas, fue el único valor con diferencia significativa $(p<0.05)$ entre rodales cosechados y no cosechados (Fig. 2). No obstante que en rodales cosechados de Avicennia se observa una distribución de individuos por categoría diamétrica ligeramente más continua que aquella presente en rodales no cosechados (Fig. 3), los modelos de distribución diamétrica para rodales cosechados (ec. 5) y no cosechados (ec. 6) fueron estadísticamente iguales, ya sea mediante la prueba de paralelismo $(F=0.18, p>0.68$, $n=36)$ o interacción $(F=0.35, p>0.56, n$ $=36$ ):

$$
\begin{aligned}
y & =540.285-162.529 \ln (x) \\
R^{2} & =0.45 \\
F & =10.72 \\
p & =0.0060 \\
y & =698.071-213.711 \ln (x) \\
R^{2} & =0.42 \\
F & =9.52 \\
p & =0.0087
\end{aligned}
$$

Así mismo, no se encontraron diferencias significativas $(p>0.05)$ entre rodales cosechados y no cosechados de esta especie, al tomar en cuenta los valores promedio del numero de individuos y área basal por categoría diamétrica (Fig. 3 y 4).
Los valores promedio de densidad en Avicennia también se ajustaron a una distribución Weibull, tanto para rodales no cosechados (ec. 7) como cosechados (ec. 8), habiendo obtenido la estimación más robusta de los parámetros con el procedimiento de momentos no centrales. La abundancia de la repoblación natural es mostrada por categoría de altura, tanto para rodales cosechados como no cosechados, en la figura 5 para Laguncularia y en la figura 6 para Avicennia.

Las abundancias totales y de la clase de altura 1 de Laguncularia fueron mayores, en promedio, para los rodales cosechados en comparación con los no cosechados; la clase de altura 2 presentó un valor superior en los rodales no cosechados, y se tuvieron cantidades similares de individuos en la clase 3 de ambos rodales (Fig. 5). Aunque las diferencias anteriores no fueron significativas $(p>0.05)$, se observó que al menos una clase de altura estuvo presente en los cinco sitios cosechados, en contraste con sólo dos sitios no cosechados que mostraron repoblación natural (Fig. 5).

Los valores de abundancia promedio de renuevos en Avicennia fueron, a diferencia de la especie anterior, mayores para los rodales no cosechados en las tres clases de altura que para los cosechados. Asimismo, se distinguió la presencia de al menos una clase de altura en los cinco sitios no cosechados, en comparación con tres de los sitios cosechados que no mostraron individuos. Sin embargo, solamente en la clase 3 la cantidad promedio de renuevos fue significativamente diferente $(p<0.05)$ entre rodales cosechados y no cosechados (Fig. 6).

$$
\begin{aligned}
& f(x)=\frac{0.8083}{2.5094}\left(\frac{x-3.6444}{2.5094}\right)^{0.8083-1} \exp \left[-\left(\frac{x-3.6444}{2.5094}\right)^{0.8083}\right] \\
& f(x)=\frac{0.8046}{2.8423}\left(\frac{x-3.8087}{2.8423}\right)^{0.8046-1} \exp \left[-\left(\frac{x-3.8087}{2.8423}\right)^{0.8046}\right]
\end{aligned}
$$



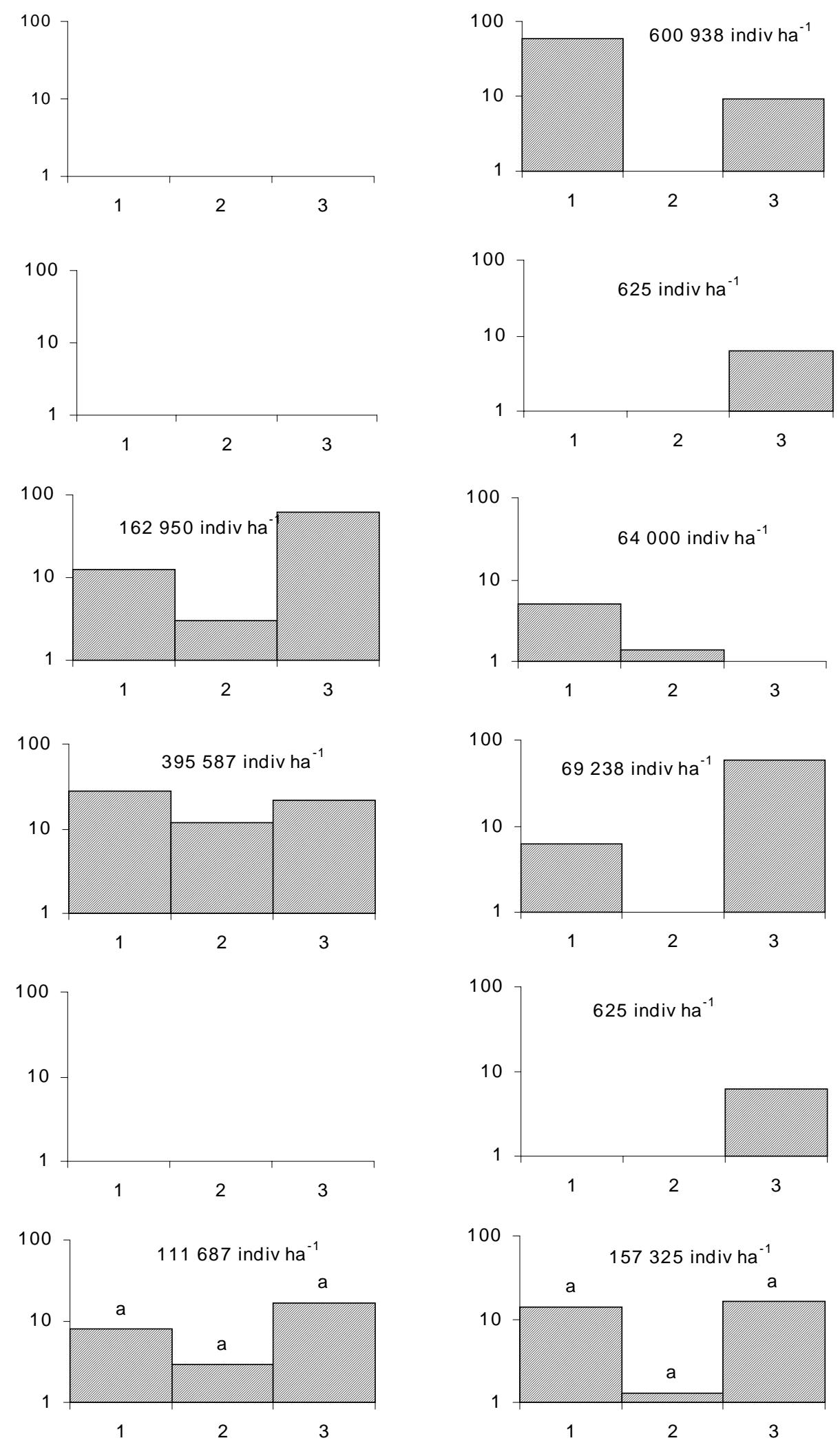

Figura 5. Distribución de la regeneración natural (escala logarítmica) por clases de altura en rodales no cosechados y cosechados de Laguncularia racemosa en el Ejido Villa

Juárez. Clase 1: plantas $<30 \mathrm{~cm}$ altura, clase $2: 30 \mathrm{~cm}<$ plantas $<1.3 \mathrm{~m}$ altura y clase $3: 1.3 \mathrm{~m}<$ plantas $<2.5$ $\mathrm{cm}$ DAP. Letras iguales indican diferencias no significativas $(p>0.05)$ entre rodales cosechados y no cosechados. 

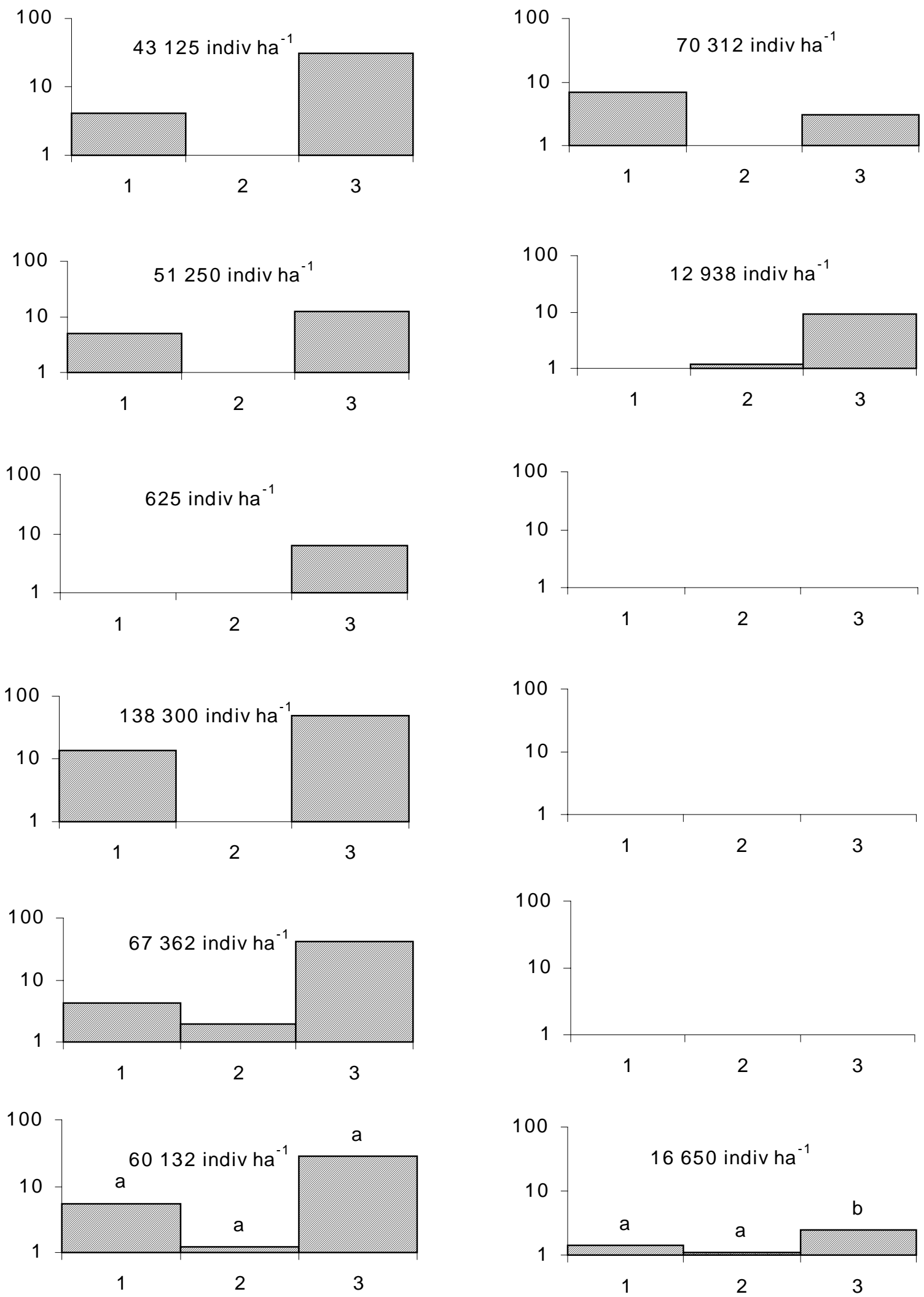

Figura 6. Distribución de la regeneración natural (escala logarítmica) por clases de altura en rodales no cosechados y cosechados de Avicennia germinans en el Ejido Villa Juárez. Clase 1: plantas $<30 \mathrm{~cm}$ altura, clase 2: $30 \mathrm{~cm}<$ plantas $<1.3 \mathrm{~m}$ altura y clase $3: 1.3 \mathrm{~m}<$ plantas $<2.5 \mathrm{~cm}$ DAP. Letras iguales indican diferencias no significativas $(p>0.05)$ entre rodales cosechados y no cosechados. 
Cuando el número de renuevos en la clase 3 se incluyó como categoría diamétrica, los modelos de distribución de la densidad en Laguncularia tuvieron un mejor ajuste, tanto para rodales no cosechados (ec. 9) como cosechados (ec. $10)$, en comparación con aquellos donde no se incluye la regeneración (ver ec.1 y 2); sin embargo, los modelos que consideran renuevos también fueron estadísticamente iguales entre sí con base en las pruebas de paralelismo $(F=0.17, p$ $>0.68, n=38)$ e interacción $(F=0.22, p$ $>0.64, n=38$ ):

$$
\begin{aligned}
& y=1424.81-427.881 \ln (x) \\
R^{2}= & 0.71 \\
F= & 41.16 \\
p= & 0.0001 \\
& y=1258.49-381.410 \ln (x) \\
R^{2}= & 0.62 \\
F= & 27.59 \\
p= & 0.0001
\end{aligned}
$$

En la misma forma, cuando el número de renuevos en la clase 3 se incluyó como categoría diamétrica, los modelos de distribución de la densidad en Avicennia tuvieron un mejor ajuste, tanto para rodales no cosechados (ec. 11) como cosechados (ec. 12), en comparación con aquellos donde no se incluye la regeneración (ver ec. 5 y 6); sin embargo, los modelos que comprenden renuevos también fueron estadísticamente iguales entre sí de acuerdo con las pruebas de paralelismo $(F=1.31, p>0.26, n=38) \mathrm{e}$ interacción $(F=3.27, p>0.08, n=38)$ :

$$
y=1575.9-508.334 \ln (x)
$$

$R^{2}=0.59$

$F=24.72$

$p=0.0001$

$$
\begin{aligned}
& y=409.38-115.572 \ln (x) \\
R^{2}= & 0.43 \\
F= & 12.69 \\
p= & 0.0024
\end{aligned}
$$

\section{DISCUSIÒN}

Debido a que no se cuenta con información publicada respecto a la influencia de la extracción de madera en la estructura de los manglares de otras regiones de México o del mundo, resulta difícil comparar los datos obtenidos en el presente estudio, particularmente aquellos relacionados con el ajuste de las distribuciones diamétricas del número de individuos a la función Weibull.

La escasa información que se tiene está referida a un patrón logarítmico negativo de distribución diamétrica para las especies Avicennia bicolor en Costa Rica (Jiménez, 1990) y $A$. marina en Papua Nueva Guinea (Robertson et al., 1991) y Pakistán (Saifullah, 1994). Este patrón de distribución diamétrica, el cual también fue encontrado para los manglares de Laguncularia y Avicennia en el presente trabajo, es comúnmente aludido por Rollet (1978) para describir la estructura de bosques tropicales en diversas partes del mundo, y es mencionado por Bongers et al. (1988) como un indicador de que la población tiene una buena reproducción y una constante incorporación de individuos.

Con excepción de la densidad y área basal de Laguncularia pertenecientes a la categoría diamétrica de $18 \mathrm{~cm}$, no se detectaron diferencias estadísticas significativas en la estructura de rodales cosechados y no cosechados para los manglares en el área de estudio. Sin embargo, y no obstante la ausencia de diferencias significativas en la cantidad de repoblación natural entre rodales cosechados y no cosechados de Laguncularia, se tuvo una presencia más continua de la repoblación en rodales cosechados de esta especie; situación que puede 
atribuirse a un mejoramiento en las condiciones de establecimiento de las plantas (p.e. mayor incidencia de luz) (Smith, 1987), derivado de aperturas en el dosel (Hartshorn, 1989).

En contraste con lo antes mencionado, la abundancia de repoblación en Avicennia fue menor y más irregular en rodales cosechados que en los no cosechados. Al comparar con otros estudios, la repoblación de esta especie en rodales cosechados fue también menor que lo citado para $A$. marina en Qatar (17 300 plantas/ha) por Abdel-Razik (1991) y en Pakistán (47 350 plantas/ha) por Saifullah et al. (1994). Esta abundancia puede deberse a los bajos niveles de extracción de árboles y de área basal efectuados en rodales de esta especie, en comparación con aquellos aplicados a rodales de Laguncularia, que tal vez no permitieron una apertura suficiente del dosel para el establecimiento de la repoblación como se ha encontrado para $A$. marina en Australia (Clarke y Allaway, 1993).

Sin embargo, y como se ha mencionado para otras regiones del mundo, la escasa repoblación en Avicennia probablemente sea debida al daño de propágulos y plántulas por cangrejos (Smith, 1987a; Osborne y Smith, 1990) o por desecación de éstas (McKee, 1995; Patterson et al., 1997). Por tanto es requerida mayor investigación en este aspecto. Del mismo modo, y con la finalidad de establecer niveles adecuados de cosecha de madera que utilicen en todo su potencial los manglares analizados, se propone la realización de estudios adicionales sobre crecimiento en diámetro (árboles) y altura (repoblación), así como la determinación de tasas de incorporación y mortalidad de individuos a través de sus diferentes clases de altura y categorías diamétricas.

\section{CONCLUSIONES}

Los valores en la distribución del número de árboles y área basal por categoría diamétrica en los rodales de manglar cosechados, no fueron en general significativamente diferentes de aquellos obtenidos en los rodales no cosechados. De igual manera, las cantidades de repoblación natural registradas por categoría de altura para rodales de manglar cosechados, tampoco fueron en general significativamente diferentes de aquellas obtenidas para rodales no cosechados. No obstante lo anterior, se tuvo una mayor presencia y distribución menos discontinua de la repoblación en rodales cosechados de Laguncularia que en los no cosechados, en contraste con la repoblación de Avicennia, la cual fue menos abundante y más irregular en los rodales cosechados.

\section{RECONOCIMIENTOS}

A la comunidad del Ejido Villa Juárez, su ayuda en el registro de la información de campo, al Ing. Alfonso Solís, su apoyo en la conformación de la base de datos, al Dr. Humberto Vaquera y M.C. Miguel Acosta, su ayuda en el análisis estadístico, a los revisores externos de Madera y Bosques, sus comentarios y sugerencias y al Programa para el Desarrollo Forestal-Nayarit, su respaldo económico para este trabajo.

\section{REFERENCIAS}

Abdel-Razik, M.S. 1991. Population structure and ecological performance of the mangrove Avicennia marina (Forssk.) Vierh. on the Arabian Gulf coast of Qatar. Journal of Arid Environments 20 (3):331-338. 
Alrasjid, H. y R. Effendi. 1986. Pengaruh penebangan terhadap kerusakan permudaan alam hutan mangrove di kelompok hutan sungai Sepada, Kalimantan Barat (The effect of felling to the natural regeneration damage of mangrove forest in Sepada river forest complex West Kalimantan). Buletin Penelitian Hutan 476:1-22.

Bongers, F.; J. Popma; J. Meave del Castillo y J. Carabias. 1988. Structure and floristic composition of the lowland rain forest of Los Tuxtlas, Mexico. Vegetatio 74:55-80.

Cintron, G.; A.E. Lugo y R. Martinez. 1985. Structural and functional properties of mangrove forests. In: W.G. D'Arcy y M.D. Correa A., eds. The botany and natural history of Panama. Monographs in Systematic Botany from the Missouri Botanical Garden 10:53-66.

Clarke, P.J. y W.G. Allaway. 1993. The regeneration niche of the grey mangrove (Avicennia marina): effects of salinity, light and sediment factors on establishment, growth and survival in the field. Oecologia 93:548-556.

Clutter, J.L.; J.C. Fortson; L.V. Pienaar; G.H. Brister y R.L. Bailey. 1983. Timber management: a quantitative approach. John Wiley \& Sons. Nueva York, EUA. 333 p.

Flores V., F.J.; F. González F.; D. Segura Z. y P. Ramírez G.. 1992. Mangrove ecosystems of the Pacific coast of Mexico: Distribution, structure, litterfall, and detritus dynamics. In: V. Seeliger, ed.. Coastal plant communities of Latin America. Academic Press. Nueva York, EUA. pp. 269-288.

García, E. 1987. Modificaciones al sistema de clasificación climática de Köppen. 4a ed. México, D.F. 217 p.
Hartshorn, G.S. 1989. Application of gap theory to tropical forest management: natural regeneration on strip clearcuts in the Peruvian Amazon. Ecology 70 (3):567-569.

INEGI (Instituto Nacional de Estadística, Geografía e Informática). 1993. Anuario estadístico del estado de Nayarit. Gobierno del Estado de Nayarit. México. 308 p.

INEGI (Instituto Nacional de Estadística, Geografía e Informática). 1994. Anuario estadístico del estado de Nayarit. Gobierno del Estado de Nayarit. México. 340 p.

INEGI (Instituto Nacional de Estadística, Geografía e Informática). 1996. Santiago Ixcuintla, Estado de Nayarit; cuaderno estadístico municipal edición 1995. México. 148 p.

INEGI (Instituto Nacional de Estadística, Geografía e Informática). 1997. Nayarit; Conteo de población y vivienda 1995; Perfil sociodemográfico. México. 104 p.

INEGI (Instituto Nacional de Estadística, Geografía e Informática). 1998. Anuario estadístico del estado de Nayarit. Gobierno del Estado de Nayarit. México. 454 p.

Instituto de Geofísica/UNAM. 1997. Tablas de predicción de mareas. Puertos del Océano Pacífico. Servicio Mareográfico Nacional. México. $415 p$.

Jiménez, J.A. 1988. The dynamics of Rhizophora racemosa Meyer forests on the Pacific coast of Costa Rica. Brenesia 30:1-12.

Jiménez, J.A. 1990. The structure and function of dry weather mangroves on the Pacific coast of Central America, with emphasis on Avicennia bicolor forests. Estuaries 13 (2):182-192. 
Lahmann, E.J.; S.C. Snedaker y M.S. Brown. 1987. Structural comparisons of mangrove forests near shrimp ponds in southern Ecuador. Interciencia 12 (5):240-243.

López Portillo, J.A. 1982. Ecología de manglares y de otras comunidades de halófitas en la costa de la Laguna de Mecoacán, Tabasco. Tesis Profesional. Facultad de Ciencias, UNAM. México, D.F. 160 p.

Lot, A. y A. Novelo. 1990. Forested wetlands of Mexico. In: A.E. Lugo; M. Brinson y $S$. Brown., eds. Forested wetlands. Ecosystems of the world 15. Elsevier. Amsterdam, Holanda. pp. 287-298.

Lot H., A.; C. Vázquez Y. y F. Menéndez L. 1975. Physiognomic and floristic changes near the northern limit of mangroves in the Gulf Coast of Mexico. In: G.E. Walsh; S.C. Snedaker y H.J. Teas, eds. Proceedings of International Symposium on Biology and Management of Mangroves. vol. 1:8-11 October 1974. East-West Centre, Honolulu, Hawaii. Institute of Food and Agricultural Sciences. University of Florida, Gainesville, Florida. EUA. pp:52-61.

Magaña T., O.S.; J.M. Torres R. y M. Acosta M. 1992. Guía de uso de West: Programa para estimar parámetros de la función de distribución de probabilidades Weibull. Campo Experimental Valle de México. INIFAP. Chapingo, México. 29 p.

McKee, K.L. 1995. Seedling recruitment patterns in a Belizean mangrove forest: effects of establishment ability and physico-chemical factors. Oecologia 101:448-460.

Menéndez L., F.J. 1976. Los manglares de la Laguna de Sontecomapan, Los Tuxtlas, Ver.; estudio florístico-ecológico. Tesis Profesional. Facultad de Ciencias, UNAM. México, D.F. 115 p.
Osborne, K. y T.J. Smith III. 1990. Differential predation on mangrove propagules in open and closed canopy forest habitats. Vegetatio 89:1-6.

Patterson, S.; K.L. McKee y I.A. Mendelssohn. 1997. Effects of tidal inundation and predation on Avicennia germinans seedling establishment and survival in a subtropical mangal/ salt marsh community. Mangroves and Salt Marshes 1:103-111.

Pool, D.J.; S.C. Snedaker y A.E. Lugo. 1977. Structure of mangrove forests in Florida, Puerto Rico, México and Costa Rica. Biotropica 9 (3):195-212.

Ramírez A., M.C. 1995. Estructura y demografía del manglar del estero La Angostura, Laguna Oriental, Oax. y su relación con algunos parámetros ambientales. Tesis Profesional. Facultad de Estudios Superiores Zaragoza. UNAM. México, D.F. 73 p.

Robertson, A.I.; P.A. Daniel y P. Dixon. 1991. Mangrove forest structure and productivity in the Fly River estuary, Papua New Guinea. Marine Biology 111 (1):147-155.

Rollet, B. 1978. Description, functioning and evolution of tropical forest ecosystems. 5. Organization. In: UNESCO. Tropical forest ecosystems (Natural resources research XIV). Paris, France. pp. 112-142.

Saifullah, S.M.; S.S. Shaukat y S. Shams. 1994. Population structure and dispersion pattern in mangroves of Karachi, Pakistan. Aquatic Botany 47:329-340.

SAS Institute Inc. 1996. JMP IN ${ }^{\circledR}$ Version 3.1.5 for Windows.

Seber, G.A.F. 1977. Linear regression analysis. John Wiley \& Sons. Nueva York, EUA. 465 p. 
Silva, C.A.R.; L.D. Lacerda; L.F.F. Silva y C.E. Rezende. 1991. Forest structure and biomass distribution in a red mangrove stand in Sepetiba Bay, Rio de Janeiro. Revista Brasileira de Botanica 14(1):21-26.

Smith III, T.J. 1987. Effects of light and intertidal position on seedling survival and growth in tropical tidal forests. Journal of Experimental Marine Biology and Ecology 110:133-146.

Smith III, T.J. 1987a. Effects of seed predators and light level on the distribution of Avicennia marina (Forsk.) Vierh. in tropical, tidal forests. Estuarine, Coastal and Shelf Science 25:43-51.

SPP (Secretaría de Programación y Presupuesto). 1981. Síntesis geográfica y anexo cartográfico de Nayarit. México, D.F. 224 p.

Srivastava, P.B.L. y K. Daud. 1978. Progress of natural regeneration after final felling under the current silvicultural practices in Matang Mangrove Reserve. Pertanika 1 (2):126-135.

Sukardjo, S. 1987. Natural regeneration status of commercial mangrove species (Rhizophora apiculata and Bruguiera gymnorrhiza) in the mangrove forest of Tanjung Bungin, Banyuasin District, South Sumatra. Forest Ecology and Management 20:233-252.
Thom, B.G. 1967. Mangrove ecology and deltaic geomorphology: Tabasco, Mexico. Journal of Ecology 55:301-343.

Torres R., J.M.; M. Acosta M. y O.S. Magaña T. 1992. Métodos para estimar los parámetros de la función Weibull y su potencial para ser predichos a través de atributos de rodal. Agrociencia serie Recursos Naturales Renovables 2(2):57-76.

Trejo T., J.C.; R. Durán e I. Olmsted. 1993. Manglares de la península de Yucatán. In: S.I. Salazar V. y N.E. González, eds.. Biodiversidad Marina y Costera de México. CONABIO/ CIQRO. México, D.F. pp. 660-672.

Valdez H., J.I. 1991. Estructura fisonómica del bosque de mangles de la Laguna de Agua Brava, Nayarit. Tesis Profesional. División de Ciencias Forestales. UACH. Chapingo, Estado de México. 263 p.

Vázquez Y., C. 1971. La vegetación de la Laguna de Mandinga, Veracruz. Anales del Instituto de Biología de la Universidad Nacional Autónoma de México, Serie Botánica 42 (1):49-94.

Velázquez M., A.; H.M. de los Santos P. y J.I. Valdez H. 1995. Producción forestal de México. VII Censo Agropecuario, 1991. Instituto Nacional de Estadística, Geografía e Informática. Colegio de Postgraduados en Ciencias Agrícolas. México. 170 p.»

1 Programa Forestal. Instituto de Recursos Naturales. Colegio de Postgraduados. Km 36.5 Carr. MéxicoTexcoco. Montecillo, Estado de México. 56230. c.e.: ignaciov@colpos.mx.

Manuscrito recibido el 27 de febrero de 2002.

Aceptado el 4 de abril de 2002.

Este documento se debe citar como:

Valdez H., J.I. 2002. Aprovechamiento forestal de manglares en el estado de Nayarit, costa Pacífica de México. Madera y Bosques Número especial:129-145. 